\title{
Perivascular Hematoma along Pulmonary Artery Complicating Aortic Dissection
}

\author{
Hiroaki Wakakuri, Hideya Hyodo, Toshihiko Ohara and Masahiro Yasutake \\ Department of General Medicine and Health Science, Nippon Medical School, Tokyo, Japan
}

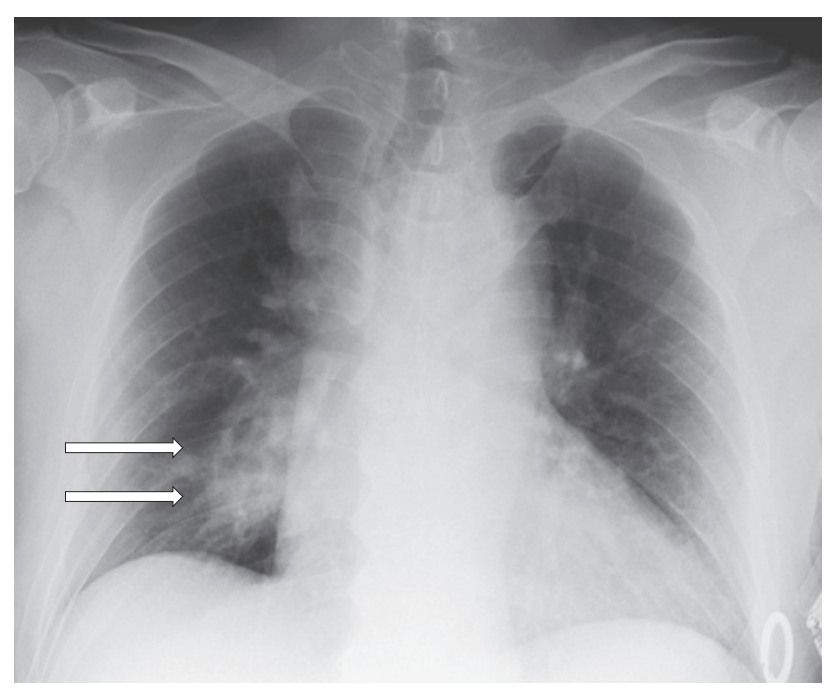

Fig. 1

\section{Case Presentation}

A 54-year-old man presented to our general medicine clinic with acute upper back pain. On examination, blood pressure was $90 / 46 \mathrm{~mm} \mathrm{Hg}$ in the right arm and 133/100 mm Hg in the left arm. A chest X-ray film revealed dilatation of the upper mediastinum and opacity in the right lung (Fig. 1). A subsequent contrast-enhanced computed tomography examination demonstrated Stanford type A aortic dissection (Fig. 2A) with hemorrhage from the ruptured false lumen extending along the right pulmonary artery (PA) (Fig. 2B, Fig. 2C) consistent with the radiographic opacity. The patient underwent emergent ascending aorta replacement surgery.

Perivascular hematoma along the PA is an infrequent complication of acute type A aortic dissection, although the prevalence of this complication has been reported, in one retrospective study, to be $9 \%$. Radiographic manifestations of perivascular hematoma mimicking other hilar abnormalities have also been reported ${ }^{2-4}$. Hemorrhage from a ruptured false lumen can easily extend to the adventitia surrounding the pulmonary trunk and the main PA, because this adventitia is shared at the root of the great vessels. Hemorrhage may result in narrowing of the lumina of the major pulmonary arteries owing to the low pressure of arteries within the lung ${ }^{5}$. In the present case, 50\% luminal stenosis of the right PA was observed. Clinicians should bear in mind the likelihood of this complication in the setting of an abnormal hilar shadow on chest X-ray films in patients with suspected aortic dissection.

Conflict of Interest: The authors declare no conflict of interest.

Correspondence to Hiroaki Wakakuri, MD, Department of General Medicine and Health Science, Nippon Medical School, 1-1-5 Sendagi, Bunkyo-ku, Tokyo 113-8603, Japan 


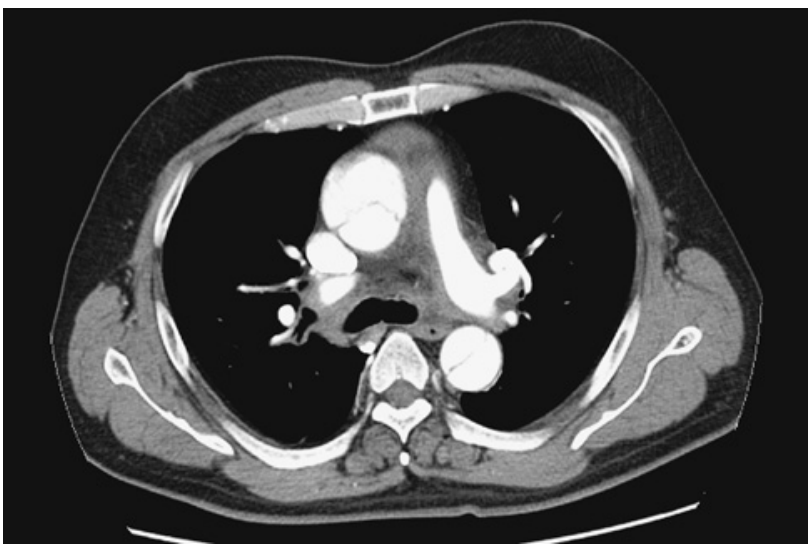

Fig. 2A

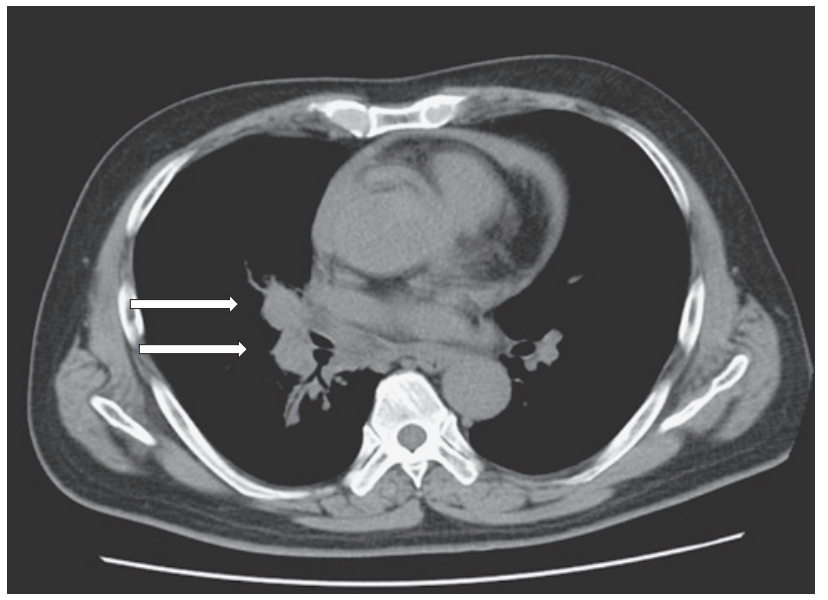

Fig. 2C

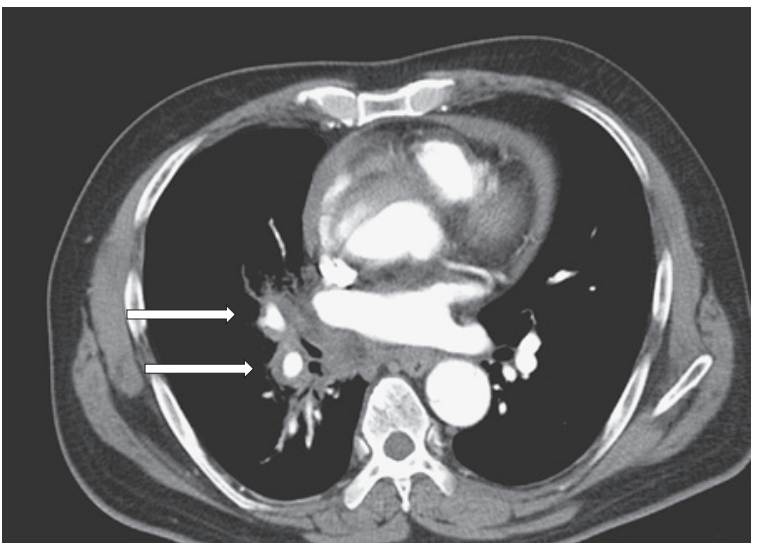

Fig. 2B

Fig. 1 Chest X-ray film demonstrating opacity of the right lung (arrows).

Fig. 2A Contrast-enhanced computed tomography of the chest.

Fig. 2B Contrast-enhanced computed tomography of the chest demonstrating hematoma extension along the pulmonary artery.

Fig. 2C Unenhanced computed tomography of the chest demonstrating the area of pulmonary artery hemorrhage (arrows).

\section{References}

1. Sueyoshi E, Matsuoka Y, Sakamoto I, Uetani M: CT and clinical features of hemorrhage extending along the pulmonary artery due to ruptured aortic dissection. Eur Radiol 2009; 19: 1166-1174.

2. Colice GL, Lenz J, Schned A: Unilateral hyperlucent lung due to interstitial pulmonary hemorrhage from aortic dissection. Am J Medicine 1989; 86: 250-252.

3. Charnsangavej C: Occlusion of the right pulmonary artery by acute dissecting aortic aneurysm. AJR Am J Roentgenol 1979; 132: 274-276.

4. Nasrallah A, Goussous Y, El-Said G, Garcia E, Hall RJ: Pulmonary artery compression due to acute dissecting aortic aneurysm: Clinical and angiographic diagnosis. Chest 1975; 67: 228-230.

5. Roberts WC: Aortic dissection: Anatomy, consequences, and causes. Am Heart J 1981; 101: 195-214. 